\title{
The MeerKAT International GHz Tiered Extragalactic Exploration (MIGHTEE) Survey
}

\author{
Matt J. Jarvis $^{* 1,2}$, A. R. Taylor ${ }^{2,3,4}$, I. Agudo ${ }^{5}$, James R. Allison ${ }^{6}$, R. P. Deane ${ }^{7}$, B. \\ Frank $^{3,4}$, N. Gupta ${ }^{8}$, I. Heywood ${ }^{1,7}$, N. Maddox ${ }^{9}$, K. McAlpine $^{2,10}$, Mario G. Santos ${ }^{2}$, A. \\ M. M. Scaife ${ }^{11}$, M. Vaccari ${ }^{2,12}$, J. T. L. Zwart ${ }^{3}$, E. Adams ${ }^{9}$, D. J. Bacon ${ }^{13}$, A. J. Baker ${ }^{14}$, \\ Bruce. A. Bassett ${ }^{15,16,17}$, P. N. Best ${ }^{18}$, R. Beswick ${ }^{11}$, S. Blyth ${ }^{3}$, Michael L. Brown ${ }^{11}$, M. \\ Brüggen $^{19}$, M. Cluver ${ }^{2}$, S. Colafranceso ${ }^{20}$, G. Cotter ${ }^{1}$, C. Cress ${ }^{2,21}$, R. Davé2,16, C. \\ Ferrari $^{22}$, M. J. Hardcastle ${ }^{23}$, C. Hale ${ }^{1}$, I. Harrison ${ }^{11}$, P. W. Hatfield ${ }^{1}$, H.-R. Klöckner ${ }^{24}$, \\ S. Kolwa ${ }^{2,25}$, E. Malefahlo ${ }^{2}$, T. Marubini ${ }^{2}$, T. Mauch ${ }^{10}$, K. Moodley ${ }^{26}$, R. Morganti ${ }^{9}$, R. \\ Norris $^{6,27}$, J. A. Peters ${ }^{1}$, I. Prandoni ${ }^{12}$, M. Prescott ${ }^{2}$, S. Oliver ${ }^{28}$, N. Oozeer ${ }^{10,15,29}$, H. J. \\ A. Röttgering ${ }^{30}$, N. Seymour ${ }^{31}$, C. Simpson ${ }^{32}$, O. Smirnov ${ }^{7}$, D. J. B. Smith ${ }^{21}$, K. \\ Spekkens $^{33}$, J. Stil ${ }^{34}$, C. Tasse ${ }^{7,35}$, K. van der Heyden ${ }^{3}$, I. H. Whittam ${ }^{2}$, W. L. \\ WIIliams ${ }^{21}$ \\ ${ }^{1}$ University of Oxford \& ${ }^{2}$ University of the Western Cape \\ E-mail: matt.jarvis@physics.ox.ac.uk
}

\begin{abstract}
The MIGHTEE large survey project will survey four of the most well-studied extragalactic deep fields, totalling 20 square degrees to $\mu \mathrm{Jy}$ sensitivity at Giga-Hertz frequencies, as well as an ultra-deep image of a single $\sim 1 \mathrm{deg}^{2}$ MeerKAT pointing. The observations will provide radio continuum, spectral line and polarisation information. As such, MIGHTEE, along with the excellent multi-wavelength data already available in these deep fields, will allow a range of science to be achieved. Specifically, MIGHTEE is designed to significantly enhance our understanding of, (i) the evolution of AGN and star-formation activity over cosmic time, as a function of stellar mass and environment, free of dust obscuration; (ii) the evolution of neutral hydrogen in the Universe and how this neutral gas eventually turns into stars after moving through the molecular phase, and how efficiently this can fuel AGN activity; (iii) the properties of cosmic magnetic fields and how they evolve in clusters, filaments and galaxies. MIGHTEE will reach similar depth to the planned SKA all-sky survey, and thus will provide a pilot to the cosmology experiments that will be carried out by the SKA over a much larger survey volume.
\end{abstract}

MeerKAT Science: On the Pathway to the SKA,

25-27 May, 2016,

Stellenbosch, South Africa

${ }^{*}$ Speaker. 


\section{Introduction}

The MeerKAT International GHz Tiered Extragalactic Exploration (MIGHTEE) survey is a project being undertaken by an international collaboration of researchers to explore cosmic evolution by creating deep images of the $\mathrm{GHz}$ radio emission in continuum, spectral line and polarisation. The survey will be conducted over $20 \mathrm{deg}^{2}$ of the best studied regions of the extragalactic sky observable from the southern hemisphere, namely COSMOS, XMM-LSS, ECDFS and ELAIS-S1. The nominal sensitivity will be $\sim 1 \mu \mathrm{Jy}$ over the full bandwidth of $900-1670 \mathrm{MHz}$, at a resolution of $\sim 6$ arcsec, with additional observations made over a smaller area with the S-band receiver. In the following we outline the key science aims of MIGHTEE.

\section{Science Goals}

\subsection{MIGHTEE-LADUMA synergy: The cosmic evolution of $\mathrm{HI}$}

The MIGHTEE HI survey component and the LADUMA HI survey (Baker et al. these proceedings) can be thought of as two tiers of a survey 'wedding cake' at L-band. The $\sim 20$ square degree MIGHTEE survey will form the wide, shallow tier out to intermediate redshifts $(\mathrm{z} \sim 0.5)$ and LADUMA the narrow, deep tier $(\mathrm{z}<1.4)$. LADUMA will include a deep L-band component plus a deep UHF-band component. The two surveys are therefore highly complementary in that MIGHTEE will observe a larger volume at low redshift and will gather a larger sample of low redshift galaxies, while for the overlapping L-band component, LADUMA's extreme depth will probe lower HI masses, and LADUMA's UHF-band component will cover redshifts inaccessible to MIGHTEE. There is substantial overlap in the science goals of the two surveys, notably in probing the HI mass function (HIMF) and cosmic neutral gas density over a range of cosmic time and different environments. At low redshifts MIGHTEE will observe more galaxies at the high-mass end of the HIMF due to the larger local cosmological volume probed and LADUMA, due to its $\sim 4.5 \mathrm{x}$ higher sensitivity in the L-band, will detect more low HI mass galaxies. By combining $\mathrm{HI}$ detections from the two surveys we will be able to measure both the low- and high-mass ends of the HIMF, significantly reducing the associated statistical errors due to low source counts, out to intermediate redshift (Fig. 1; 48).

\subsection{From the gas to the stars: the onset of star formation over cosmic time}

Galaxies follow known scaling relations, such as the so-called star-formation (SF) main sequence (39) relating SF with stellar mass. Underlying these relations is a complex cycle of acquisition, storage, consumption, expulsion and re-acquisition of gas acting to regulate a galaxy's ability to form stars (e.g. 17). The vastly different evolution of cosmic SF density and HI density from $z \sim 1$ to the present day implies a complex, non-linear relation between the two processes. State-of-the-art simulations which attempt to incorporate a neutral gas component into galaxies are unable to reproduce the detailed distribution of Hi content in galaxies. In particular, they do not exhibit the mass-dependent relation between HI mass and stellar mass, found to be at least partly due to dark matter halo angular momentum (47). This mismatch between observations and simulations indicates missing physics in our galaxy formation prescriptions that can only be brought to light with large samples of $\mathrm{HI}$ measurements spanning orders of magnitude in stellar mass. 

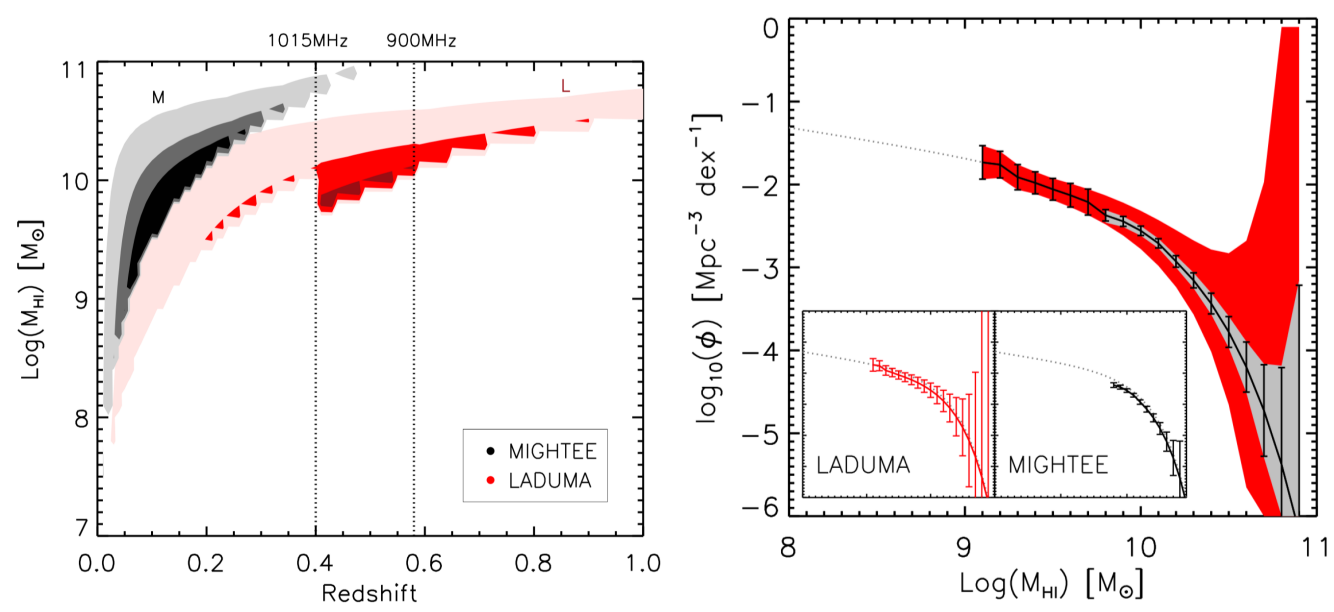

Figure 1: (left) The expected coverage of the $\mathrm{HI}$ mass versus redshift plane by combining the LADUMA and MIGHTEE surveys. The grey shaded regions show the MIGHTEE coverage and the red shaded regions show the LADUMA coverage. (right) The expected constraints on the HI mass function. It is clear that MIGHTEE is required to measure the high-mass end whereas LADUMA pins down the low-mass end at $z<0.5$. Taken from (48).

Furthermore, while the stellar properties of galaxies spanning the full range of masses over a large range of redshifts have been well studied, our knowledge of the neutral gas (HI) content of these same galaxies is restricted to the local universe. Surveys such as ALFALFA (23) cover large areas of sky and include a variety of cosmic environments, but do not have the frequency coverage required to explore a range of redshifts. If we hope to understand the build-up of stellar mass, we must observe the neutral gas reservoir of fuel from which the molecular gas forms, eventually turning into stars, along with the interface between this gas and the galaxies and environment in which it is located. MIGHTEE will open up this new parameter space in the study of the neutral gas component of galaxies.

MIGHTEE will have the sensitivity and frequency coverage required to detect $\mathrm{HI}$ in a statistically significant number of $\mathrm{M}_{\mathrm{HI}}^{\star}$ galaxies at $z=0.2$, and will detect the rare, most HI-massive galaxies to $z \sim 0.5$, due to the large volume probed. Thus, together with LADUMA, MIGHTEE will revolutionise our understanding of the HI content of galaxies over a cosmologically significant redshift range, as a function of stellar mass and environment.

MIGHTEE not only provides us with the ability to trace the origins of the gas that eventually turns into stars, but with the exquisite sensitivity to radio-continuum emission, we will also measure the end-point, namely the star-formation rate (SFR). Radio-continuum observations closely trace the far-infrared emission of galaxies at all redshifts, the so-called Far-infrared-radio correlation (e.g. 35; 64), as such they offer a unique method of measuring the evolution of the SFR density over cosmic time, free of dust-obscuration, a key issue in sensitive UV-based surveys (see e.g. 46). Furthermore, deep far-IR and sub-mm surveys are generally confusion limited at SFR $100 \mathrm{M}_{\odot} \mathrm{yr}^{-1}$ (e.g. 54; 21), and although it provides high angular resolution, ALMA cannot survey large areas efficiently (18). At the depth of the MIGHTEE survey, we will be able to detect galaxies with $\mathrm{SFR} \sim 15 \mathrm{M}_{\odot} \mathrm{yr}^{-1}$ to $z=1$ and $\mathrm{SFR} \sim 150 \mathrm{M}_{\odot} \mathrm{yr}^{-1}$ to $z \sim 3$. By using the excellent existing 
optical and near-IR data we are able to trace the evolution of SFR in galaxies as a function of mass, colour, environment etc, thus gaining critical insight of which galaxies form the bulk of the stars, in what environments and when.

\subsection{The galaxy merger history from $\mathrm{OH}$ megamasers}

The global SFR and the assembly of massive elliptical galaxies are inextricably connected to how galaxies merge over cosmic time. Recently merged (luminous and ultra-luminous infrared) galaxies provide the perfect conditions for detecting Hydroxyl $(\mathrm{OH})$ megamasers, which are often found within $1 \mathrm{kpc}$ of heavily dust-obscured AGN (e.g. 14). OH megamasers are therefore ideal luminous radio beacons $\left(L_{\mathrm{OH}} \sim 10^{4} L_{\odot}\right)$ for tracing the merger history of the Universe (e.g. 10). The Arecibo $\mathrm{OH}$ Megamaser survey (14), which detected 52 masers out to $z=0.23$, represents the current state-of-the-art in our understanding of the nearby megamaser population and the luminosity function (15). The MIGHTEE survey will provide a unique opportunity to carry out a deep blind search for $\mathrm{OH}$ megamaser emission (and $\mathrm{OH}$ absorption) between $z=0$ and 0.85 . The low-redshift luminosity function of (15), would imply a detection yield of $\sim 10 \mathrm{OH}$ megamasers. However, this number is highly dependent on the evolution of the galaxy merger rate as a function of redshift, often parameterised as $(1+z)^{m}$ with $m \approx 2-8$, which could easily lead to an order of magnitude more in MIGHTEE. With MIGHTEE we will measure the merger rate traced by $\mathrm{OH}$ megamasers directly.

\subsection{Quenching: the role of environment}

One of the key unknowns in models of galaxy evolution is how SF in galaxies becomes quenched. Environmentally-related processes such as ram-pressure stripping and strangulation, where the cold and/or hot-gaseous haloes are removed due to the density of the medium through which a galaxy is moving, can explain some of the required quenching. However, internal processes such as SF and AGN-driven winds may also play an important, if not critical, role (e.g. 56). Furthermore, it is clear from both semi-analytic models and hydro-dynamical simulations (e.g. 20) and observations (e.g. 27;30) that these internal feedback processes are also dependent on the mass of the galaxy or halo in which they reside.

Recent work $(28 ; 16 ; 29)$ using optical and near-infrared data has shown that the quenching of low-mass galaxies in the environments of large massive galaxies, which trace the largest dark matter haloes, does not appear to be strongly related to their position in the halo. This suggests that quenching is an internal process, with the environment playing a lesser role. However, when the massive tracer galaxy is strongly star forming, then the satellite galaxies are less quenched. Thus, the process is not simple and requires an understanding of the evolution of haloes over time, and also an indication of the time at which the halo itself formed. Furthermore, it is now clear that the so-called "Galactic Conformity" (see e.g. 42; 31), where neighbouring haloes appear to be related, continues to high $(z>2)$ redshift (29).

The depth of MIGHTEE allows us to directly observe where and when the cold gas is being stripped from galaxies through the sensitivity to $\mathrm{HI}$, and the areal coverage provides enough cosmic volume to study such physical processes in the densest and sparsest environments. With the same set of observations, and following the prescription outlined in (29), we will relate the prevalence of 
quenching to the halo mass, and the proximity to the centre of the halo, and to the galaxies which may accelerate quenching such as those exhibiting AGN activity.

\subsection{AGN fueling and feedback}

It is widely thought that AGN activity may be responsible for switching off SF in massive galaxies, or at least maintaining a "quenched" state (Section 2.4) once the SF has terminated. However, a direct observational link between AGN activity and SF at high redshifts remains elusive. Recent studies from both a theoretical (63) and observational (40) perspective have shown that powerful radio-loud AGN may actually provide a positive form of feedback. On the other hand, there is little evidence for any type of feedback from radio-quiet objects based on studies using Herschel (e.g., 9), with recent studies suggesting that the host galaxies of radio-quiet AGN are similar to the general galaxy population (e.g. 58).

\subsubsection{Tracing the mechanical feedback from AGN jets}

To understand AGN feedback and the interplay between SF and AGN activity, both in the AGN host and the wider environment, a survey is required that spans enough cosmological volume to include the rare powerful AGN at $z \sim 1$, but with a depth that detects SFGs at similar redshifts to the AGN. Radio is arguably the best line of attack due to the sensitivity to both SF activity and AGN activity. Given that different forms of AGN feedback are invoked in current semi-analytic and hydrodynamic models of galaxy formation (e.g. 33), it is essential that we understand such processes if we are to understand the evolution of galaxies in general.

Observational evidence (e.g., 6) suggests that many or most low-power $\left(\mathrm{P}<10^{25} \mathrm{~W} \mathrm{~Hz}^{-1}\right)$ radio galaxies in the local universe (the numerically dominant population) correspond to a distinct type of AGN. These sources accrete through a radiatively inefficient mode (the so-called "radio mode"), rather than the radiatively efficient accretion mode typical of radio-quiet AGN selected at optical or X-ray wavelengths (sometimes called 'quasar mode'; see (32) for a recent review covering these feedback processes). The role of these two accretion modes appears to be strongly influenced by the environment (e.g., 69) while the level of radio-jet activity appears to be a strong function of the stellar mass of the host galaxy (e.g. 34; 74).

Therefore, deeper radio surveys that cover enough area of sky with the best multi-wavelength data are required to probe the evolution of these relationships and the accretion mode dichotomy over cosmic time; this is key information for any attempt to incorporate radiative and mechanical feedback from radio-loud AGN in models of galaxy, group and cluster formation and evolution.

The depth and breadth of MIGHTEE will enable unique studies of the entire AGN population from $z \sim 0-6$, providing a complete view of nuclear activity in galaxies and its evolution, unbiased by gas/dust selection effects. If current simulations and measurements are correct, we will detect a factor of $\sim 20$ more low-accretion rate radio sources at $z>1$ than the current VLA-COSMOS data $(65 ; 66 ; 67)$, due to the greater depth and increased area. This dramatic increase means that we will accurately measure the evolution of such sources to $z \sim 2$, testing the key ingredient of galaxy evolution simulations. Furthermore, this will allow the amount of energy deposited into the intergalactic medium by such objects to be measured over the era when such sources are active, thus providing the key input to the evolution of mechanical feedback from AGN jets. 


\subsection{2 $\mathrm{H}$ I as a direct probe of neutral gas accretion and feedback in AGN}

MIGHTEE will provide a homogeneous survey of 21-cm H I absorption and emission from AGN across the radio luminosity function, enabling a direct investigation into the symbiotic relationship between AGN activity and neutral gas in galaxies. The H I gas content of AGN has received increasing attention in recent years, both through emission and absorption studies. H I emission probes the global properties of the neutral gas, such as mass, velocity structure and neutral gas fraction. This offers a direct measurement of the large-scale fuel source for the AGN, and a probe of triggering mechanisms. Complementary to this, H I absorption seen against the radio continuum source adds vital information on the state and kinematic behaviour of neutral gas within the central regions of radio-loud AGN (e.g. 22).

Existing samples for both types of study are limited in size, with previous work based on relatively shallow observations of pre-selected (and possibly biased) galaxy samples. MIGHTEE will provide a unique opportunity to revolutionise this field through a deep, wide-area dataset, with an unbiased sample of radio-selected AGN and a rich suite of ancillary data not normally available in wider 21-cm surveys.

Using both direct $\mathrm{H} \mathrm{I}$ emission detections, and stacking analyses, the $\mathrm{H}$ I gas content of samples of galaxies selected to display AGN activity will be compared against samples of non-AGN matched in mass, environment, and other properties. This will allow the investigation of the origin of the AGN activity and the influence of the AGN on its host galaxy. At the sensitivity of MIGHTEE, it will be possible to detect $\mathrm{H}$ I masses down to around $2 \times 10^{9} \mathrm{M}_{\odot}$ out to $z=0.2$ at the $5 \sigma$ level, assuming typical line widths of $250 \mathrm{~km} \mathrm{~s}^{-1}$. This is a substantial advance in sensitivity over previous studies, and should allow detection of the H I gas in the AGN hosts, given typical gas fractions of $10 \%$ (e.g. 19). There will be around 1000 AGN of all classes hosted by galaxies more massive than $10^{10} \mathrm{M}_{\odot}$ in the MIGHTEE area.

For absorption studies, assuming a typical line width of $100 \mathrm{~km} \mathrm{~s}^{-1}$, we expect to obtain a sample of approximately 240 radio galaxies brighter than $1 \mathrm{mJy}$ within the comoving volume bounded by $z_{\mathrm{HI}} \approx 0.58$. Approximately 50 of these sources will be brighter than $10 \mathrm{mJy}$ and we will be sensitive to cold gas clouds with peak opacities greater than $1 \%$ and typical column densities greater than $2 \times 10^{20} \mathrm{~cm}^{-2}$. This sample spans the radio luminosity function between $10^{23}<L_{1.4} / \mathrm{W} \mathrm{Hz}^{-1}<10^{25}$, allowing us to directly observe and test evolutionary models of neutral gas accretion in low-excitation radio galaxies (e.g. 70). The brightest radio galaxies in our absorption sample will also enable a study of jet-mode feedback in neutral gas (e.g. 53) out to intermediate cosmological redshifts, and is a natural complement to the MeerKAT Absorption Line Survey (MALS; 24).

\subsection{AGN polarisation as a probe of environment}

Radio-loud AGN are intrinsically highly polarised; the fractional polarisation in high-resolution, high-frequency images can approach the theoretical maximum of $\sim 70 \%$ (57).

MIGHTEE will provide extremely sensitive, broad-band L-band observations, supplemented in some cases with S-band data as described in Section 3.1, and so will give measurements of integrated or moderately resolved polarisation as a function of frequency for many thousands of radio-loud AGN, allowing samples to be subdivided by e.g. luminosity and redshift to measure the 
evolution of intrinsic and environmental properties. Among other projects, MIGHTEE's polarisation surveys, coupled with total intensity information and optical identifications/redshifts for the target sources, will allow us to (i) statistically relate observed (de)polarisation to source environment, by combining with optical and (where possible) X-ray data available in the target fields; (ii) investigate the dependence of observed (de)polarisation on physical size, testing the prediction of models that sources observed at low resolution become more polarised as they become physically larger, so that the average Faraday depth to/dispersion in front of the lobes becomes smaller (25); it does indeed appear to be the cases that sources of larger angular size are more polarised in deep surveys (59) but MIGHTEE will give much larger samples and constrain the physical size scale on which this takes place, and hence the physical size of the hot-gas halo; (iii) carry out largescale statistical studies of the Laing-Garrington effect for a large number of moderately resolved AGN, probing physical conditions in the centre of the host environment and giving a prior for every source on the angle to the line of sight; and (iv) carry out a statistical test of the (13) depolarisation law and hence probe the fine structure and possibly the power spectrum of the magnetic field in the intra-cluster medium (ICM).

\subsection{Large scale structure \& cosmology}

Covering $20 \mathrm{deg}^{2}$, MIGHTEE will provide the ideal data set to pave the way for large-scale cosmology experiments with the SKA (see $1 ; 37 ; 61$ ).

\subsubsection{An accurate measurement of the evolution of bias}

The depth and breadth of MIGHTEE will allow us to measure the bias, i.e. how radio sources of different types (e.g. star-forming galaxies, Fanaroff-Riley Class I and II, etc), trace the underlying dark-matter distribution (e.g. 44; 4; 49). Carrying this out over the best multi-wavelength fields allows us to accurately disentangle the different types of sources, whilst obtaining the required number density (apart from very rare FRIIs). Furthermore, cross-correlations with the optical/nearinfrared has been shown to be a powerful technique to measure the bias of rarer sources (45). Such measurements of the bias can then be used in the wider area surveys where it is much more difficult to measure, due to a lack of multi-wavelength and redshift information. Indeed, these measurements will be needed in order to carry out large cosmological tests with the proposed all-sky SKAMID survey, as it would be at a similar depth to MIGHTEE. Figure 2 shows the current state of the art in the measurement of the clustering of radio sources to high-redshift using the most recent JVLA-COSMOS data, and the expected constraints with a single $7.5 \mathrm{deg}^{2}$ MIGHTEE field. The error ellipse on the amplitude and the slope is improved by around an order of magnitude.

\subsubsection{Weak lensing}

The coherent distortion of distant galaxy shapes due to gravitational light deflection by large scale structure ("weak lensing" or "cosmic shear") is recognised as one of the most powerful probes of dark matter and dark energy, and is a major cosmology science goal for the SKA $(11 ; 26 ; 8)$.

MIGHTEE does not have the resolution to measure shapes of galaxies and thus cannot directly measure the shear for weak lensing measurements. However, by conducting the survey over the fields with the best optical data (which have been and continue to be used for weak lensing measurements), we are able to use the integrated polarisation vector from MIGHTEE data, to inform 

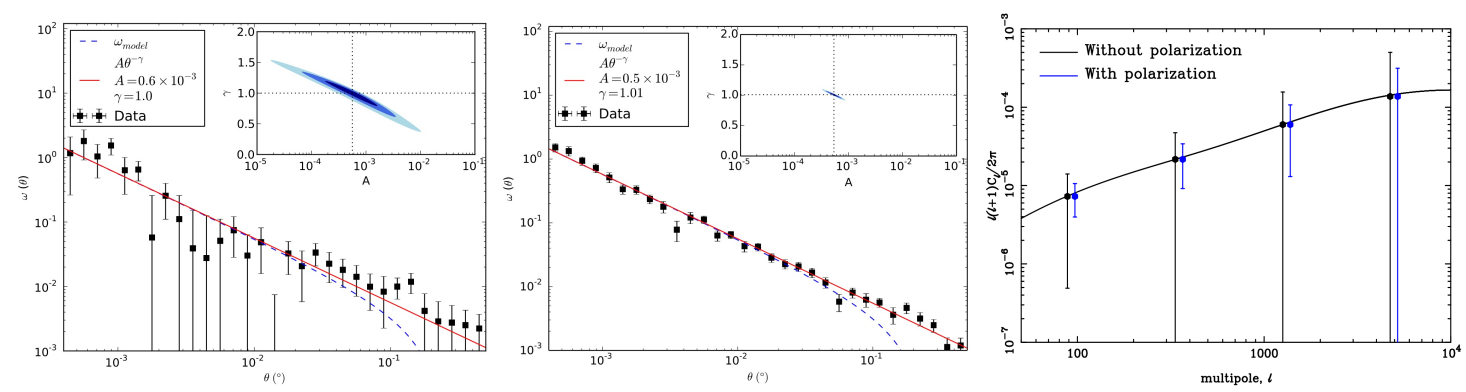

Figure 2: (left) The measured two-point correlation function of radio sources in the recent $\sim 1.5 \mathrm{deg}^{2}$ JVLA-COSMOS 3GHz survey (PI: Smolčić), with an equivalent $5 \sigma$ depth of $S_{1.4} \sim 20 \mu \mathrm{Jy}$ (Hale et al. submitted). (center) The expected constraints on the two-point correlation function for MIGHTEE with $S_{1.4}=5 \mu \mathrm{Jy}$, for a single $7.5 \mathrm{deg}^{2}$ field. (right) Forecasts for a measurement of the cosmic shear weak lensing power spectrum using MIGHTEE polarisation observations combined with galaxy shape measurements from overlapping optical surveys. For comparison we show forecasts for the same sample of galaxies without the polarisation information. We assume we can obtain useful polarisation measurements for $\sim 5 \%$ of those galaxies detected in total intensity, and we assume a scatter of $\sim 10$ degrees in the relation between the polarisation orientation and the disk structure of the source galaxies.

on the intrinsic alignment of the galaxies before they are lensed. This removes one of the most significant systematics in weak lensing surveys, and if proven would allow MIGHTEE to be the path finder for weak lensing with polarisation for the SKA.

Our forecasts suggest that MIGHTEE polarisation data combined with shape information from best overlapping optical data, will provide a $\sim 3.5 \sigma$ detection of the cosmic shear effect (Fig. 2; right). This would represent the first detection of weak lensing using the polarisation technique.

\subsubsection{Cosmic magnetism and large-scale structure: The magnetic cosmic web}

MIGHTEE will provide a supremely-dense Rotation Measure (RM) grid - an effect caused by the interaction of a magneto-ionic medium, with the linearly polarised synchrotron emission from background or embedded cluster galaxies - and permit measurement of the properties of magnetic fields embedded in the large-scale structure of the universe. While clusters contain hot plasma of $T>10^{7} \mathrm{~K}$, filaments are expected to be filled with plasma of $10^{7} \mathrm{~K}>\mathrm{T}>10^{5} \mathrm{~K}$, referred to as the Warm Hot Intergalactic Medium (WHIM). The plasmas may be magnetised; diverse processes for seed magnetic fields have been suggested, and the seed fields can be further amplified through compression and turbulent dynamos as well as leakage of galactic media during the hierarchical structure formation in the universe (60).

Simulations predict that the inter-galactic magnetic field (IGMF) in filaments would induce excess Faraday Rotation Measures with a flat second-order structure function of $\sim 100 \mathrm{rad}^{2} \mathrm{~m}^{-4}$ for angular separation of $r \gtrsim 0.1^{\circ}$. The power from this contribution to the RM structure function will be distinguishable from the foreground of the Milky Way galaxy on angular scales smaller than a few degrees. An RM data set with a sky density of several 100 to 1000 polarised sources per square degree, and with RM precision of $\sim 1 \mathrm{rad} \mathrm{m}^{-2}$ is required to accurately reconstruct the structure function of RM variance due to the IGMF in the cosmic web (3). MIGHTEE will probe this structure function with the required number density and RM precision, and over angular scales 
of a few arcminutes to several degrees, offering our best opportunity before the SKA to use this technique to detect the magnetic cosmic web.

\subsubsection{Resolving Massive Galaxies out to Cosmological Distances: Dark Matter in Galaxies}

MIGHTEE's combination of high sensitivity and angular resolution for HI produces a column density sensitivity $\left(\sim 1 \mathrm{M}_{\odot} / \mathrm{pc}^{2}\right.$ over $\delta_{V}=20 \mathrm{~km} / \mathrm{s}$ at $\left.\mathrm{S} / \mathrm{N}=3\right)$ that is sufficient to resolve the $\mathrm{HI}$ distributions of HI-rich galaxies out to cosmological distances. MIGHTEE will therefore be the first survey to probe the distribution of dark and luminous matter in a statistical sample of massive galaxies beyond the local volume. The tight correlation between HI mass and HI diameter for local galaxies (73), coupled with the expected number of HI detections using the local HIMF (51), implies that MIGHTEE will resolve $>150$ galaxies with $\mathrm{M}_{\mathrm{HI}}>10^{10} \mathrm{M}_{\odot}$ at redshifts $0.07<z<$ 0.12. This is sufficient to estimate the HI distribution and rotation curve shape by modelling the 3D HI cubes and optical images (41). MIGHTEE is the only planned HI survey capable of resolving a significant sample of massive galaxies out to cosmological distances before the advent of SKA1, and highly complementary to samples at lower redshifts that will be produced by wide-field $\mathrm{HI}$ surveys with the Australian Square Kilometre Array Pathfinder (ASKAP; 38) and (Apertif; 72).

\subsubsection{Low-mass, nearby galaxies and the satellite problem}

Predictions based on $\Lambda \mathrm{CDM}$ excel at matching observations of large scale structure. However, on small scales, the effects of baryonic physics are important and it can be difficult to match simulations to observations. Observations of low-mass galaxies offer constraints on the implementation of baryonic physics, and are especially valuable as they include spatially resolved kinematic information. Systems with HI masses below $\sim 10^{8} \mathrm{M}_{\odot}$ are especially important for testing our understanding of baryonic physics and $\Lambda \mathrm{CDM}$. The HI kinematics of galaxies in this mass range place them in dark matter halos incompatible with expectations from abundance matching results (55). The lowest mass systems (below $\sim 2 \times 10^{7} \mathrm{M}_{\odot}$ ) are extremely rare; in the full ALFALFA survey there are only $\sim 70$. Finding and studying these lowest mass galaxies is critical for understanding at what mass range and due to which processes dark matter halos stop hosting observable galaxies. With MIGHTEE we expect to detect $\sim 270$ sources with $\mathrm{M}_{\mathrm{HI}}<10^{8} \mathrm{M}_{\odot}$, with $\sim 15$ of those below $10^{7} \mathrm{M}_{\odot}$. Using the HI mass-diameter relation (73), we estimate that $\sim 7$ of the sources below $10^{8} \mathrm{M}_{\odot}$ will be resolved with at least three MeerKAT beams, and one to two sources may be resolved with five beams.

\subsection{Cluster radio halos and relics}

Clusters of galaxies show radio emission on a very broad range of scales, from discrete sources associated with AGN to diffuse emission on Mpc scales. The latter points to the existence of a non-thermal component (cosmic rays and magnetic fields) of the ICM. Diffuse radio sources at the cluster center are generally known as "radio halos", while elongated polarised Mpc-scale radio structures at the cluster periphery are known as "radio relics". Current models indicate that Mpc-scale diffuse radio emission in the form of radio halos traces the turbulent re-acceleration of particles due to merger events, whereas relics are the result of electron acceleration and magnetic field compression resulting from ICM shocks (e.g. 12). 
The angular resolution and excellent brightness sensitivity provided by MIGHTEE will probe radio emission on all scales relevant for cluster radio emission to high redshift. MIGHTEE's multiwavelength coverage will be crucial in shedding light on the origin of the non-thermal cluster components, as detailed multi-wavelength analyses of clusters are essential to properly characterise the physical differences between "radio-loud" and "radio-quiet" systems. This kind of study is crucial to understand, for instance, why not all merging clusters host diffuse radio sources, or viceversa (e.g. 7), and the role played by gravitational and non-gravitational effects on the evolution of the ICM.

\subsection{Galaxy clusters and their magnetic fields}

Galaxy clusters are the largest known magnetised structures in the universe and therefore are unique laboratories to investigate the origin of large-scale magnetic (B) fields. In spite of the wealth of evidence for the existence of ICM magnetic fields, measurements of both the field strengths and morphologies of clusters are still scattered within the literature. Information on the cluster B-fields can be derived from detailed images of extended radio emission in clusters (radio halos, mini-halos and relics; 71) and through Faraday Rotation measure synthesis. With MIGHTEE we will be able to measure the magnetic field properties of hundreds of galaxy clusters in the MIGHTEE cosmic volume. The Faraday rotation provides a clean measure of the magnetic field strength along the line of sight for uniform fields, and the magnetic field strength directly for turbulent fields (43).

Constraining the behaviour of magnetic fields in galaxy clusters is not only important for studies of the intra-cluster medium itself, but can also be used to constrain the origin of cosmic magnetic fields more widely by examining the evolution of cluster fields as a function of redshift. Early-type seed fields are expected to produce a characteristic evolution in cluster magnetic field properties when combined with expectations of turbulent amplification. The high density and precision of the MIGHTEE RM grid will offer the best opportunity before SKA to constrain the redshift evolution of cluster magnetic fields, and provide the first test of the early-type model for the origin of cosmic magnetic fields.

\subsection{The emergence and evolution of magnetic fields in galaxies}

Through its ability to detect polarisation of sources at high redshift, MIGHTEE will be a cornerstone for investigating the evolution of magnetic fields in galaxies. For disk galaxies, this range is $z \lesssim 2.5$, and for AGN and starbursts it reaches out to $z \lesssim 7$, into the epoch of reionisation. Over this period, galaxies formed and evolved, converting most of their gas into stars. The evolution of plasma and magnetic fields in galaxies is expected to be closely related to the evolution of the cosmic SFR, and tied to the intergalactic medium through accretion, galactic winds, tidal and ram stripping, and AGN activity. While $\mu \mathrm{G}$ strength magnetic fields on kpc scales may have formed in galaxy disks by $z \sim 3$, ordering on the scale of a galaxy may have taken until $z \sim 0.5$, depending on galaxy mass $(5 ; 50)$. Competing with this, galaxy interactions and continuous feedback by supernovae and stellar winds can enhance the turbulent component of the magnetic field, and drive outflows that transport plasma and magnetic field from the disk into the halo. Since these processes scale with the global SFR, significant evolution is expected between $z \sim 2$ and the present. Also, the density of Faraday rotating plasma will gradually decrease over time as galaxies transform a significant fraction of their gaseous mass into stars, implying a gradual evolution in Faraday depth. 
Based on models by (68) for spiral galaxies at $z=0$, applied to normal SF galaxies in the SKADS Simulated Skies simulation (75; 76), at the sensitivity of the MIGHTEE deep commensal polarisation image of the LADUMA field we expect $\sim 5000$ galaxies per square degree above $10 \sigma$ detected to $z \sim 3$. For the wider area survey we will detect several hundred galaxies per square degree in polarisation out to $z \sim 1$. The analysis of (68) has shown that the integrated polarisation properties of these distant galaxies can be used to detect and characterise both the large-scale and turbulent magnetic fields in galaxy disks. As shown in Figure 3, the combined L- and S-band data set is critical to this study as the structure of the depolarisation of the signal as a function of frequency over this broad band is required to reveal the magnetic properties. The MIGHTEE data set will provide probes of the emergence and evolution of magnetic fields in $\sim 10,000$ galaxies out to $z>3$.
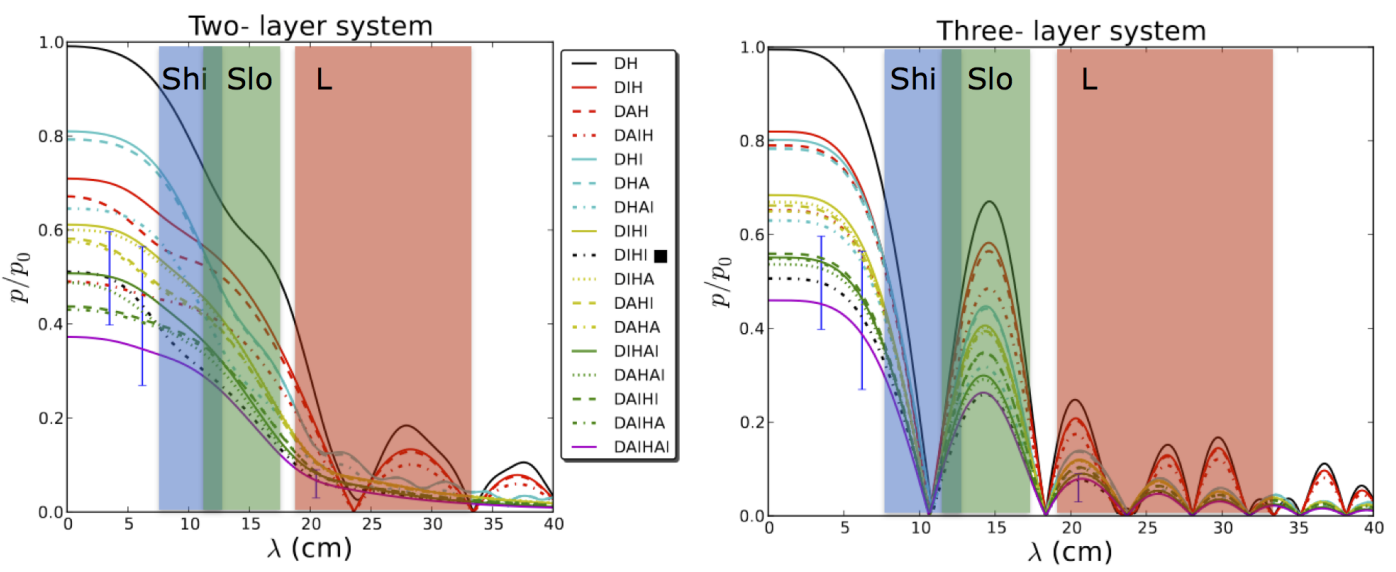

Figure 3: Models of the polarised intensity of integrated emission from galaxy disks as a function of frequency. The frequency coverages of the MeerKAT L-band and S-band are shown. Together these bands span the frequency range over which depolarisation becomes important, and both are critical to measure the depolarisation signature. The change in polarised emission is due to internal Faraday effects from the magnetic field and thermal plasma in the galaxy. Broad-band integrated spectro-polarisation data thus probes the evolution of both magnetic and thermal gas (ionisation) properties. Depolarization models are from (62).

\subsection{Digging into the noise with MIGHTEE}

Classical analyses of radio survey data relies on the direct detection of the HI, continuum or polarised emission from galaxies. However, there is significant additional information in the images for the vast number of objects that produce radio signals too faint to individually reach the direct detection flux density threshold. We will be able to take advantage of this information and investigate the statistical radio properties of classes of objects to flux densities substantially below the noise floor of the images. Following techniques developed in (52) and (77) we plan to derive deep HI mass functions, and total intensity and polarised luminosity functions through a Bayesian stacking method. Information from the rich multi-wavelength data set for the MIGHTEE fields can be used as prior information in this process. For example, $\sim 200,000$ galaxies per square degree, based on the VIDEO survey data (36) to $K_{s} \sim 23.7$, would result in a total of $4 \times 10^{6}$ galaxies to stack on over all redshifts and stellar masses. While ultimately limited by confusion in total 
intensity, MIGHTEE will provide an unique opportunity: due to its high sensitivity, a large number of the MIGHTEE galaxies detected in continuum will be SFGs containing HI, and for which we can also measure their magnetic fields through the stacked polarised emission that is unaffected by confusion.

\section{MIGHTEE observations and data}

\subsection{Observing strategy}

The key science outlined above, coupled with the current and anticipated availability of multiwavelength data, lead to the mosaic pointing setups shown in Figure 4. The number of pointings and the areas (calculated without including the area beyond the half power point of the perimeter pointings) are provided in the caption. Not shown is the COSMOS field which will be observed with a single (or tightly dithered) pointing. Using the current measurements of the MeerKAT system temperature, with $16 \mathrm{~h}$ per pointing a depth of $2 \mu \mathrm{Jy}_{\text {beam }}{ }^{-1}$ (thermal + confusion noise) will be achieved in the full-band mosaics, and a typical depth of $90 \mu \mathrm{Jy} \mathrm{beam}^{-1}$ will be reached in the $26 \mathrm{kHz}$ channels for the spectral line component of the survey. We note that one of the E-CDFS pointings will be that of the MIGHTEE-DEEP tier commensal with LADUMA.

The S-band component will cover $4 \mathrm{deg}^{2}$ in E-CDFS and $1.5 \mathrm{deg}^{2}$ in COSMOS (we do not plan to survey XMM-LSS in S-band due to satellite RFI observed in test JVLA observations). The S-band survey over a limited area of MIGHTEE will enable us to obtain a much larger bandwidth for RM synthesis, while also allowing the possibility of multi-frequency synthesis in combination with the L-band, which will help to deconfuse the L-band survey. We plan to reach a depth of $1 \mu \mathrm{Jy}_{\text {beam }}{ }^{-1}$ in the $\mathrm{S}$-band mosaics (matched to the L-band depth for a typical $\alpha=-0.7$ source), which requires $12.7 \mathrm{~h}$ per pointing (ignoring the effects of confusion, but including the overlapping pointings, and assuming a $20 \%$ sensitivity loss due to the weighting required for reliable deconvolution). Calibration overheads of $\sim 20 \%$ means that the total time required is $\sim 1920 \mathrm{~h}$.

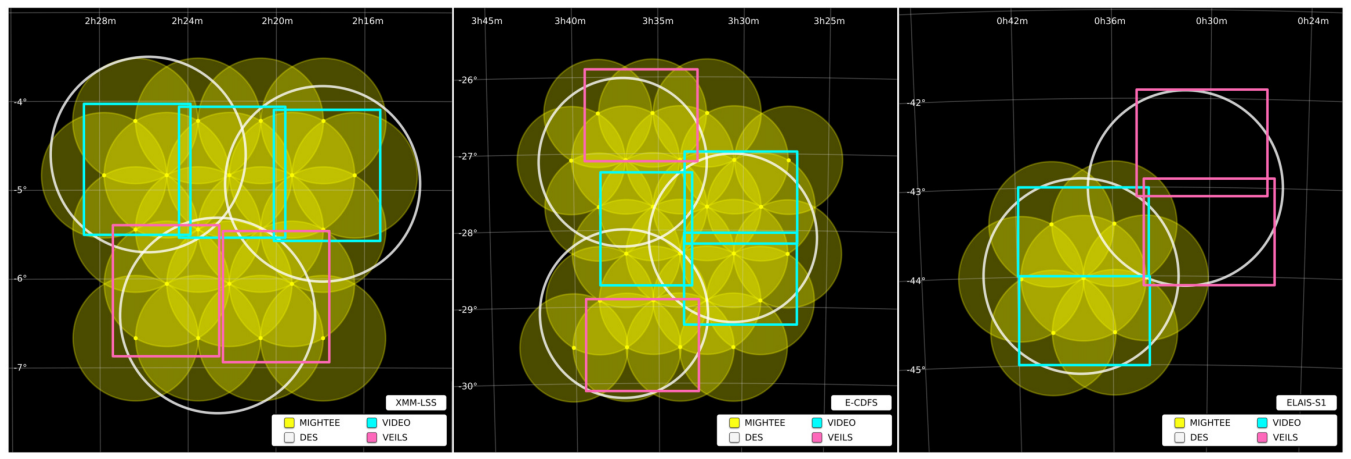

Figure 4: Current plausible pointing strategies for (left-to-right) XMM-LSS (20 pointings, $6.7 \mathrm{deg}^{2}$ ), ECDFS (24 pointings, $8.3 \mathrm{deg}^{2}$ ) and ELAIS-S1 (7 pointings, $1.6 \mathrm{deg}^{2}$ ). Not shown here is the fourth COSMOS field, which will consist of a single deep pointing. In practice the grid of E-CDFS pointings will be snapped to the LADUMA pointing centre, requiring only 23 additional pointings from MIGHTEE. 


\subsection{MIGHTEE scientific data products}

We plan to provide multiple intermediate MIGHTEE data releases of the reduced data following qualification of the data products for scientific use. Data will be released along with ancillary data where appropriate. Visibility data will be processed into images and catalogues using the data-centric processing facility at the South African Inter-University Institute for Data Intensive Astronomy (IDIA). The data processing pipeline is under development by a coordinated development team using the IDIA cloud-based development framework. We envisage that the MIGHTEE team will provide a data release 18 months after observations of a field are completed in either L- or S-band. The release will include data cubes for spectral line work, band-averaged multi-frequency synthesis total intensity images, full-Stokes intensity and RM synthesis cubes. Continuum products will have multiple resolutions optimised for different science goals, e.g. high resolution for galaxy studies, and lower resolution for cluster halo/relic work. We plan to release these data through the IDIA systems and are also investigating plans for a mirrored archive in Europe. Final data products will be released to the MeerKAT legacy data archive.

\subsection{VLBI enhancement of MIGHTEE science}

MeerKAT's data alone will be limited to an angular resolution of a few arcseconds. The $\mu \mathrm{Jy}$ radio sources will therefore be unresolved in the MIGHTEE images. This faint population is known to consist of emission from SFG and RQ AGN. Although multi-frequency analysis can aid in distinguishing the two populations, higher resolution radio imaging would allow unambiguous distinction through morphology as well as precise measures of the relative contribution of SF and AGN activity in individual objects. Very Long Baseline observations would thus offer a tremendous enhancement to the MIGHTEE project. VLBI can also play an important role in HI absorption components of MIGHTEE, contributing unique morphological insights to the interpretation of gas inflows and outflows in particular (e.g. 53). VLBI polarimetry would not only provide detailed information on sub-kpc magnetic fields and jet physics, but also enable comparison with (and separation from) the larger scale polarisation properties to be probed by MeerKAT (e.g. 2). Over the course of the MIGHTEE survey the project team will explore the possibility of including MeerKAT along with elements of the emerging Africa VLBI Network and European antennas in complementary wide-field VLBI experiments to provide high-resolution radio images of MIGHTEE sources.

\section{References}

[1] Abdalla F. B., et al., 2015, Advancing Astrophysics with the Square Kilometre Array (AASKA14), p. 17

[2] Agudo I., et al., 2015, Advancing Astrophysics with the Square Kilometre Array (AASKA14), p. 93

[3] Akahori T., Gaensler B. M., Ryu D., 2014, ApJ, 790, 123

[4] Allison R., et al., 2015, MNRAS, 451, 849

[5] Arshakian T. G., Beck R., Krause M., Sokoloff D., 2009, A\&A, 494, 21 
[6] Best P. N., Heckman T. M., 2012, MNRAS, 421, 1569

[7] Bonafede A., et al., 2014, MNRAS, 444, L44

[8] Bonaldi A., Harrison I., Camera S., Brown M. L., 2016, MNRAS, 463, 3686

[9] Bonfield D. G., et al., 2011, MNRAS, 416, 13

[10] Briggs F. H., 1998, A\&A, 336, 815

[11] Brown M. L., et al., 2015, Advancing Astrophysics with the Square Kilometre Array (AASKA14), p. 23

[12] Brunetti G., Jones T. W., 2014, International Journal of Modern Physics D, 23, 1430007

[13] Burn B. J., 1966, MNRAS, 133, 67

[14] Darling J., Giovanelli R., 2002a, AJ, 124, 100

[15] Darling J., Giovanelli R., 2002b, ApJ, 572, 810

[16] Darvish B., Mobasher B., Sobral D., Rettura A., Scoville N., Faisst A., Capak P., 2016, ApJ, 825,113

[17] Davé R., Thompson R., Hopkins P. F., 2016, MNRAS, 462, 3265

[18] Dunlop J. S., et al., 2017, MNRAS, 466, 861

[19] Fabello S., Kauffmann G., Catinella B., Giovanelli R., Haynes M. P., Heckman T. M., Schiminovich D., 2011, MNRAS, 416, 1739

[20] Gabor J. M., Davé R., 2015, MNRAS, 447, 374

[21] Geach J. E., et al., 2013, MNRAS, 432, 53

[22] Geréb K., Maccagni F. M., Morganti R., Oosterloo T. A., 2015, A\&A, 575, A44

[23] Giovanelli R., et al., 2005, AJ, 130, 2598

[24] Gupta N., et al., 2017, preprint, (arXiv:1708.07371)

[25] Hardcastle M. J., Krause M. G. H., 2014, MNRAS, 443, 1482

[26] Harrison I., Camera S., Zuntz J., Brown M. L., 2016, MNRAS, 463, 3674

[27] Hartley W. G., et al., 2013, MNRAS, 431, 3045

[28] Hartley W. G., Conselice C. J., Mortlock A., Foucaud S., Simpson C., 2015, MNRAS, 451, 1613

[29] Hatfield P. W., Jarvis M. J., 2016, preprint, (arXiv:1606.08989) 
[30] Hatfield P. W., Lindsay S. N., Jarvis M. J., Häußler B., Vaccari M., Verma A., 2016, MNRAS, 459,2618

[31] Hearin A. P., Behroozi P. S., van den Bosch F. C., 2016, MNRAS, 461, 2135

[32] Heckman T. M., Best P. N., 2014, ARA\&A, 52, 589

[33] Hopkins P. F., 2012, MNRAS, 420, L8

[34] Janssen R. M. J., Röttgering H. J. A., Best P. N., Brinchmann J., 2012, A\&A, 541, A62

[35] Jarvis M. J., et al., 2010, MNRAS, 409, 92

[36] Jarvis M. J., et al., 2013, MNRAS, 428, 1281

[37] Jarvis M., Bacon D., Blake C., Brown M., Lindsay S., Raccanelli A., Santos M., Schwarz D. J., 2015, Advancing Astrophysics with the Square Kilometre Array (AASKA14), p. 18

[38] Johnston S., et al., 2008, Experimental Astronomy, 22, 151

[39] Johnston R., Vaccari M., Jarvis M., Smith M., Giovannoli E., Häußler B., Prescott M., 2015, MNRAS, 453, 2540

[40] Kalfountzou E., et al., 2014, MNRAS, 442, 1181

[41] Kamphuis P., Józsa G. I. G., Oh S.-. H., Spekkens K., Urbancic N., Serra P., Koribalski B. S., Dettmar R.-J., 2015, MNRAS, 452, 3139

[42] Kauffmann G., Li C., Zhang W., Weinmann S., 2013, MNRAS, 430, 1447

[43] Lawler J. M., Dennison B., 1982, ApJ, 252, 81

[44] Lindsay S. N., et al., 2014a, MNRAS, 440, 1527

[45] Lindsay S. N., Jarvis M. J., McAlpine K., 2014b, MNRAS, 440, 2322

[46] Madau P., Dickinson M., 2014, ARA\&A, 52, 415

[47] Maddox N., Hess K. M., Obreschkow D., Jarvis M. J., Blyth S.-L., 2015, MNRAS, 447, 1610

[48] Maddox N., Jarvis M. J., Oosterloo T. A., 2016, MNRAS, 460, 3419

[49] Magliocchetti M., Popesso P., Brusa M., Salvato M., Laigle C., McCracken H. J., Ilbert O., 2017, MNRAS, 464, 3271

[50] Mao S. A., et al., 2017, preprint, (arXiv:1708.07844)

[51] Martin A. M., Papastergis E., Giovanelli R., Haynes M. P., Springob C. M., Stierwalt S., 2010, ApJ, 723, 1359

[52] Mitchell-Wynne K., Santos M. G., Afonso J., Jarvis M. J., 2014, MNRAS, 437, 2270 
[53] Morganti R., Fogasy J., Paragi Z., Oosterloo T., Orienti M., 2013, Science, 341, 1082

[54] Oliver S. J., et al., 2012, MNRAS, 424, 1614

[55] Papastergis E., Giovanelli R., Haynes M. P., Shankar F., 2015, A\&A, 574, A113

[56] Peng Y.-j., et al., 2010, ApJ, 721, 193

[57] Perley R. A., Carilli C. L., 1996, in Carilli C.L., Harris D.E. ed., Cygnus A — Study of a Radio Galaxy. Cambridge University Press, Cambridge, p. 168

[58] Rosario D. J., et al., 2013, A\&A, 560, A72

[59] Rudnick L., Owen F. N., 2014, ApJ, 785, 45

[60] Ryu D., Schleicher D. R. G., Treumann R. A., Tsagas C. G., Widrow L. M., 2012, SSRs, 166, 1

[61] Santos M., et al., 2015, Advancing Astrophysics with the Square Kilometre Array (AASKA14), p. 19

[62] Shneider C., Haverkorn M., Fletcher A., Shukurov A., 2014, A\&A, 567, A82

[63] Silk J., 2013, ApJ, 772, 112

[64] Smith D. J. B., et al., 2014, MNRAS, 445, 2232

[65] Smolčić V., et al., 2017a, A\&A, 602, A1

[66] Smolčić V., et al., 2017b, A\&A, 602, A2

[67] Smolčić V., et al., 2017c, A\&A, 602, A6

[68] Stil J. M., Krause M., Beck R., Taylor A. R., 2009, ApJ, 693, 1392

[69] Tasse C., Best P. N., Röttgering H., Le Borgne D., 2008, A\&A, 490, 893

[70] Tremblay G. R., et al., 2016, Nature, 534, 218

[71] Tribble P. C., 1991, MNRAS, 250, 726

[72] Verheijen M., Oosterloo T., Heald G., van Cappellen W., 2009, in Panoramic Radio Astronomy: Wide-field 1-2 GHz Research on Galaxy Evolution. p. 10

[73] Wang J., Koribalski B. S., Serra P., van der Hulst T., Roychowdhury S., Kamphuis P., Chengalur J. N., 2016, MNRAS, 460, 2143

[74] Williams W. L., Röttgering H. J. A., 2015, MNRAS, 450, 1538

[75] Wilman R. J., et al., 2008, MNRAS, 388, 1335

[76] Wilman R. J., Jarvis M. J., Mauch T., Rawlings S., Hickey S., 2010, MNRAS, 405, 447

[77] Zwart J. T. L., Santos M., Jarvis M. J., 2015, MNRAS, 453, 1740 


\section{Author affiliations}

${ }^{1}$ Astrophysics, University of Oxford, Denys Wilkinson Building, Keble Road, Oxford OX1 3RH, UK

${ }^{2}$ Department of Physics and Astronomy, University of the Western Cape, Robert Sobukwe Road, Bellville 7535, South Africa

${ }^{3}$ Department of Astronomy, University of Cape Town, Rondebosch 7701, South Africa

${ }^{4}$ Inter-University Institute for Data Intensive Astronomy, University of Cape Town, South Africa

5 Instituto de Astrofísica de Andalucía (CSIC), Apartado 3004, E-18080 Granada, Spain

${ }^{6}$ CSIRO Astronomy and Space Science, PO Box 76, Epping NSW 1710, Australia

Department of Physics \& Electronics, Rhodes University, PO Box 94, Grahamstown, 6140, South Africa

${ }^{8}$ Inter-University Centre for Astronomy and Astrophysics, India

${ }^{9}$ ASTRON, the Netherlands Institute for Radio Astronomy, Postbus 2, 7990AA, Dwingeloo, The Netherlands

${ }^{10}$ Square Kilometre Array South Africa, Pinelands 7405, Cape Town, South Africa

${ }^{11}$ Jodrell Bank Centre for Astrophysics, School of Physics and Astronomy, The University of Manchester, Oxford Road, Manchester M13 9PL, UK

${ }^{12}$ INAF - Istituto di Radioastronomia, via Gobetti 101, 40129 Bologna, Italy

${ }^{13}$ Institute of Cosmology \& Gravitation, University of Portsmouth, Dennis Sciama Building, Portsmouth PO1 3FX, UK

${ }^{14}$ Department of Physics and Astronomy, Rutgers, The State University of New Jersey, 136 Frelinghuysen Road, Piscataway, NJ 08854-8019, USA

15 African Institute for Mathematical Sciences, 6-8 Melrose Road, Muizenberg 7945, South Africa

${ }^{16}$ Department of Maths and Applied Maths, University of Cape Town, Cape Town, South Africa

${ }^{17}$ South African Astronomical Observatory, Observatory, Cape Town, 7925, South Africa

${ }^{18}$ SUPA, Institute for Astronomy, Royal Observatory, Edinburgh EH9 3HJ, UK

${ }^{19}$ Hamburger Sternwarte, Universität Hamburg, Gojenbergsweg 112, D-21029 Hamburg, Germany

${ }^{20}$ School of Physics, University of the Witwatersrand, Private Bag 3, 2050 Johannesburg, South Africa

${ }^{21}$ CHPC, CSIR, 15 Lower Hope Rd, Rosebank, 7700, South Africa

${ }^{22}$ Laboratoire Lagrange, Université Côte d'Azur, Observatoire de la Côte d'Azur, CNRS, Blvd de l'Observatoire, CS 34229, F-06304 Nice cedex 4, France

${ }^{23}$ Centre for Astrophysics Research, School of Physics, Astronomy and Mathematics, University of Hertfordshire, College Lane, Hatfield AL10 9AB, UK

${ }^{24}$ Max-Planck-Institut für Radioastronomie Auf dem Hügel 69, 53121 Bonn, Germany

${ }^{25}$ European Southern Observatory, Karl-Schwarzschild-Str. 2, 85748, Garching, Germany

${ }^{26}$ Astrophysics \& Cosmology Research Unit, School of Mathematics, Statistics \& Computer Science, University of KwaZulu-Natal, Durban 4041, South Africa

${ }^{27}$ Western Sydney University, Locked Bag 1797, Penrith South, NSW 1797, Australia

${ }^{28}$ Astronomy Centre, Department of Physics and Astronomy, University of Sussex, Falmer, Brighton, BN1 9QH, UK

${ }^{29}$ Centre for Space Research, North-West University, Potchefstroom 2520, South Africa

${ }^{30}$ Leiden Observatory, Leiden University, P.O. Box 9513, NL-2300 RA Leiden, The Netherlands

${ }^{31}$ International Centre for Radio Astronomy Research, Curtin University, Perth, Australia

${ }^{32}$ Gemini Observatory, Northern Operations Center, 670 North AâĂŸohoku Place, Hilo, HI 96720-2700, USA

${ }^{33}$ Department of Physics, Royal Military College of Canada, PO Box 17000, Station Forces, Kingston, ON K7K 7B4, Canada

${ }^{34}$ Department of Physics and Astronomy, University of Calgary, Canada 0000-0003-2623-2064

${ }^{35}$ GEPI, Observatoire de Paris, CNRS, Universite Paris Diderot, 5 place Jules Janssen, F-92190 Meudon, France 